\title{
CORRECTING ILLUMINATION VARIATIONS IN PHOTOMICROGRAPH MOSAICS OF DAGUERREOTYPES
}

\author{
Orhan Bulan*, Robert Buckley*, Ralph Wiegandt ${ }^{\dagger}$, Gaurav Sharma* \\ *ECE Dept., University of Rochester, Rochester, NY 14627-0126 \\ ${ }^{\dagger}$ George Eastman House International Museum of Photography and Film, Rochester, NY
}

\begin{abstract}
The Cincinnati Waterfront Daguerreotype Panorama, owned by the Public Library of Cincinnati and Hamilton County, was brought to George Eastman House in 2007 for conservation treatment. At George Eastman House the plates were imaged under a microscope to capture details at nearly their full resolution and composited mosaic images comprised of 756 images per plate were created. Although the mosaic yields an otherwise unattainable contiguous high resolution and detailed view of the Cincinnati waterfront at the time of capture, non-uniformity of the capture illumination introduced a patterning that impairs the visual and analytic value of the mosaic. In this paper, we develop a mathematical model for the mosaic image capture to analyze the degradation caused by the illumination non-uniformity. Motivated by the analysis, we develop a simple signal processing solution to correct for the lighting variation by utilizing suitably tuned frequency domain filtering. Using the proposed method, we demonstrate that the patterning due to non-uniformity of illumination is corrected, resulting in a significantly improved mosaic.
\end{abstract}

Index Terms - lighting correction, enhancement, restoration, daguerreotypes, photography history

\section{INTRODUCTION}

The daguerreotype was the first commercially viable medium for image capture and photography. In 1839 Louis Jacques Mandé Daguerre discovered that a highly polished silver plate made light sensitive by iodine vapors and exposed in camera could form a direct positive image when exposed to mercury fumes. Heated mercury vapor condensed on the plate and combined with the reduced silver to form brilliant submicron light scattering particles. After fixing, or rinsing off the unreacted halide, there remained a direct positive monochromatic image with a large tonal range corresponding to the intensity of light falling on the plate in camera. As a photographic medium, the daguerreotype was inherently high resolution with the image continuously formed on the silver with no emulsion.

In 1848, daguerreotypists Fontayne and Porter made eight daguerreotypes composing a panorama of nearly three miles of the Cincinnati waterfront. This Cincinnati Waterfront Daguerreotype Panorama is now a culturally and historically significant artifact owned by the Public Library of Cincinnati and Hamilton County. It provides an extremely high resolution record and a microscope reveals details of the city waterfront at the time of the capture.

The Cincinnati Waterfront Daguerreotype Panorama was imaged under a microscope at the George Eastman House in 2007. Each of the eight 8.5-by-6.5-inch square plates forming the daguerreotype panorama was imaged on a robotic microscope stage and composited into a mosaic of 756 images. While the mosaic provided a contiguous and high resolution view of the city waterfront in 1848 , spatial nonuniformity of the capture illumination introduced an objectionable visible patterning in the mosaic. This patterning is an undesirable artifact that can be seen online in mosaiced images posted by Wired Magazine describing the work [1].

In this paper, we develop a signal processing solution for correcting for the aforementioned artifact. Using generic information regarding the capture set up, we model the mosaic image capture, obtaining a mathematical representation of the captured mosaic that comprehends the degradation introduced by the illumination nonuniformity. Analysis, using our mathematical abstraction, allows us to characterize the degradation in the frequency domain, motivating a simple signal processing correction using suitably tuned frequency domain filtering. Using the method developed, the non-uniformity of illumination is corrected, yielding a much improved mosaic. We address efficient estimation of the patterning correction by using lower resolution down-sampled versions of the overall mosaic to estimate and correct the patterning variation in the final high resolution mosaic. The signal processing techniques we apply are well-known and relatively simple. The work, however, serves to illustrate how these "textbook techniques" can help solve real-world problems and also serves as an excellent example for pedagogy, providing students with a simple application of frequency transforms that aids their intuition.

The rest of this paper is organized as follows. In Section 2, we provide background on the daguerreotype, its significance, and characteristics that make high resolution imaging desirable. We also outline specific details of how the mosaic images of the Cincinnati Waterfront Daguerreotype Panorama were captured. In Section 3, we model and analyze the image capture setting motivating our algorithm for correction of illumination non-uniformity. We consider a computationally efficient realization for processing the large size image and present sample results from the processing. Section 4 concludes the paper.

\section{BACKGROUND}

The Conservation Laboratory at the George Eastman House imaged each daguerreotype plate at 16-times magnification through a Zeiss V-12 automated stereo microscope with an integrated micropositioning stage. Each image represented a section of the daguerreotype approximately $1 / 6$ inch by $1 / 4$ inch. Zeiss AxioVision imaging software was used to automatically capture and mosaic the images to create a single image of the daguerreotype. The AxioVision software was designed primarily for biological applications and tailored for high magnification, small field of view, and fixed light path illumination for transmitted, bright-field, and darkfield modes. As a result, it was not well suited for the mosaic application at relatively low magnification, large field of view, and external light sources. Furthermore, a daguerreotype has a specular surface 
with submicron light scattering particles on a background of parallel polishing scratches that impose a diffraction effect. This meant that the inherent illumination variations could not be eliminated by the system optics or the native software.

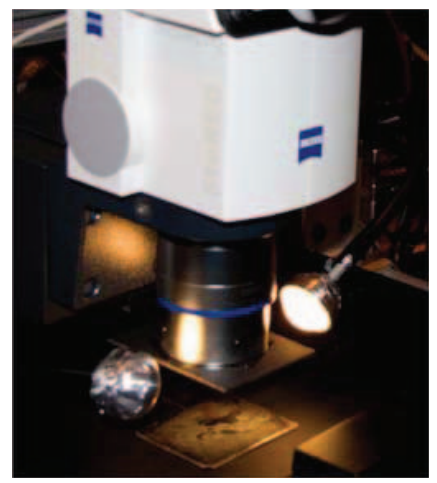

Fig. 1. Imaging set-up.

After experimenting with a number of alternative lighting arrangements, the version shown in Fig. 1 was selected because it offered the least objectionable spatial patterning. This set up uses two opposing diffuse light sources fabricated from MR16 parabolic reflector bulbs modified to insert a fiber optic light source and the hemisphere of a white ping pong ball as a diffuser. These light sources were equalized by using a "software overexposure mode" of the AxioVision to adjust their positions independently so that the opposing light sources had complementary illumination patterns across the camera field of view.

With this method of equalization, there remain variations due to a combination of the optical system and illumination profile that created a visible pattern in the mosaic image which can be seen in Fig. 2, which represents the plate 2 of the Cincinnati Waterfront Daguerreotype. The pattern is most noticeable in areas in the daguerreotype where there was a uniform flat tonal range, such as sky or water. The final mosaic image is a composition of 756 (i.e. $27 \times 28$ ) images. Though not apparent in the low resolution full mosaic due to scaling of the image for the presentation in the paper, the high resolution microscope capture allows resolution of the the extremely high level of details in the daguerreotype. The blow up in Fig. 2 illustrates this; note the text on the signboard, which corresponds roughly to a $8 \mathrm{~mm}$ $\times 6 \mathrm{~mm}$ area on the dageurreotype plate.

\section{LIGHTING VARIATION CORRECTION}

\subsection{Image Capture Model}

We begin by developing a mathematical model for the capture process used for obtaining the composited image. We represent the image on the daguerreotype by its spatial reflectance pattern $r(x, y)$, where $x$ and $y$ denote the two-dimensional spatial coordinates which span the $W \times H$ inch extent of the plate, where $W=8.5$ inches and $H=6.5$ inches. The field of view of the (captured image region) of the microscope is assumed to correspond to a $W_{0} \times H_{0}$ inch region and the spatial pattern of illumination intensity produced over this field of view is modeled as $l_{0}(\xi, \tau)$ where $\xi$ and $\tau$ denote the two-dimensional spatial coordinates in the frame of reference established by the microscope, where the axes directions and the origins are assumed to be aligned with the coordinate system used for the daguerreotype. As indicated previously, the daguerreotype plate is

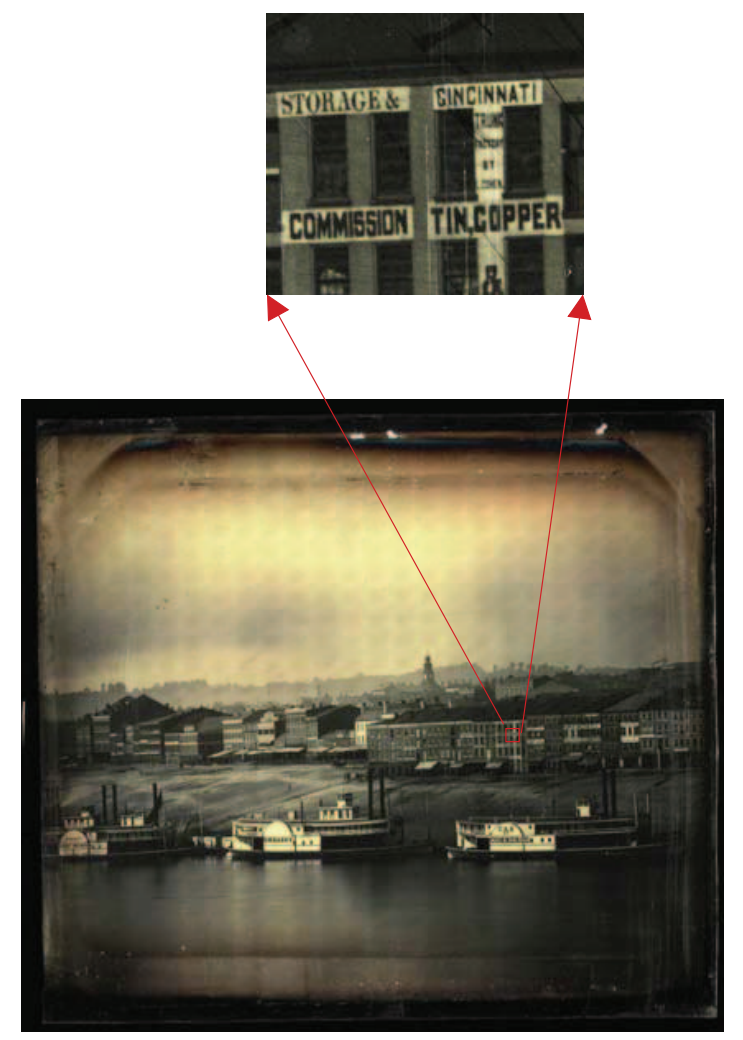

Fig. 2. Photomicrograph mosaic of plate 2 of the Cincinnati Waterfront Daguerreotype imaged at George Eastman House.

moved on the AxioVision platform, to bring different regions of the plate into the capture field of view and individual tile images corresponding to each field of view are then captured by the camera. The tiles are captured on an approximately rectilinear grid. We identify tiles by their index $(l, k)$ corresponding to the pair of indices specifying the location of the tile in this approximately rectilinear grid. The capture of an individual tile image ${ }^{1}$, say the $(l, k)^{t h}$ tile is then modeled as

$$
I_{l k}(\xi, \tau)=r\left(x_{l k}^{0}+\xi, y_{l k}^{0}+\tau\right) l_{0}(\xi, \tau),
$$

where $0 \leq \xi \leq W_{0}, 0 \leq \tau \leq H_{0}$, and $\left(x_{l k}^{0}, y_{l k}^{0}\right)$ denote the location of origin of the field of view for the $(l, k)^{t h}$ tile in the daguerreotype plate's coordinate system.

A mosaiced image $I(x, y)$ is assembled from the captured image tiles by selecting a region from each tile. The central regions that are covered by a single tile are selected from the tile, regions overlapped by more than one tile use a selection from one of the tiles ${ }^{2}$. Rewriting (1), referencing to the coordinate system of the daguerreotype, we have

$$
\left.I_{l k}\left(x-x_{l k}^{0}, y-y_{l k}^{0}\right)=r(x, y) l_{0}\left(x-x_{l k}^{0}, y-y_{l k}^{0}\right)\right),
$$

for $x \in\left[x_{l k}^{0}, x_{l k}^{0}+W_{0}\right]$ and $y \in\left[y_{l k}^{0}, y_{l k}^{0}+H_{0}\right]$. The tiled image

\footnotetext{
${ }^{1}$ Note that $l_{0}(\xi, \tau)$ is more precisely defined as the spatial pattern of intensity on encountered on the camera sensor when an ideal spatially-invariant reflecting plate with unit reflectance is imaged.

${ }^{2}$ Averaging in overlap regions can be modeled in a similar framework.
} 
can therefore be written as

$$
\begin{aligned}
I(x, y) & =r(x, y)\left[\sum_{l k} l_{l k}\left(x-x_{l k}^{0}, y-y_{l k}^{0}\right)\right] \\
& =r(x, y) l_{f}(x, y)
\end{aligned}
$$

where

$$
l_{l k}(\xi, \tau)=l_{0}(\xi, \tau) \chi_{l k}(\xi, \tau),
$$

$\chi_{l k}(\xi, \tau)$ is the region of the $(l, k)^{t h}$ tile selected for the mosaic so that $\sum_{l k} \chi_{l k}\left(x-x_{l k}^{0}, y-y_{l k}^{0}\right)=1, \forall x \in[0, W], y \in[0, H]$, and $l_{f}(x, y) \stackrel{\text { def }}{=} \sum_{l k} l_{l k}\left(x-x_{l k}^{0}, y-y_{l k}^{0}\right)$ denotes the full pattern of lighting variation across the mosaiced image, which is analyzed next.

Denote by $P_{x}$ and $P_{y}$ the nominal displacement pitches along the horizontal and vertical directions, respectively, for the rectilinear raster defining the displacements of the holding stage. The origins of the tiles are then modeled as $x_{l k}^{0}=l P_{x}+T_{k l}$, and $y_{l k}^{0}=k P_{y}+W_{k l}$, where $T_{k l}$, and $W_{k l}$ are random variables representing the positioning errors in relation to these nominal displacement pitches, which we assume are smoothly varying function of $l$ and $k$. The power spectrum of $l_{f}(x, y)$, the random pattern of illumination variation can then be analyzed by making assumptions on the statistics of the positioning errors, or by considering the case where they correspond to a slow periodic function (see, for example [2]). The analysis reveals that the power is concentrated at DC and about harmonics of the 2-D frequency $\left[1 / P_{x}, 1 / P_{y}\right]$. While space constraints do not allow the full analysis to be included here, we provide intuition for this result by considering the deterministic setting. Specifically, we assume that the $T_{k l}=W_{k l}=0, \forall k, l$ and because the displacement pitches are uniform, the selection regions are chosen as corresponding rectangles $\chi_{l k}(\xi, \tau)=\operatorname{rect}\left(\xi / P_{x}\right) \operatorname{rect}\left(\tau / P_{y}\right)$, where $\operatorname{rect}(t)=1$ for $0 \leq t \leq 1$ and 0 elsewhere. Then

$$
l_{f}(x, y)=\sum_{l k} l_{0}\left(x-l P_{x}, y-k P_{y}\right) \operatorname{rect}\left(\frac{x}{P_{x}}-l\right) \operatorname{rect}\left(\frac{y}{P_{y}}-k\right) .
$$

Now consider the signal $l_{f}^{\text {inf }}(x, y)$ obtained $^{3}$ by extending the limits of the summation for $l, k$ in (6) to $\pm \infty$. One can immediately see that $l_{f}^{\text {inf }}(x, y)$ is a periodic function with period $\left[P_{x}, P_{y}\right]$. Therefore we can write it in the form of a Fourier series as $l_{f}^{\text {inf }}(x, y)=$ $l_{0}+l^{\text {inf }}(x, y)$, where $l_{0}$ represents the 0 frequency (DC) term and $l^{\text {inf }}(x, y)$ represents the term

$$
\begin{aligned}
l^{i n f}(x, y)= & \sum_{m n} a_{m n} \cos \left(2 \pi\left[\frac{m x}{P_{x}}+\frac{n y}{P_{y}}\right]\right) \\
& +\sum_{m n} b_{m n} \sin \left(2 \pi\left[\frac{m x}{P_{x}}+\frac{n y}{P_{y}}\right]\right),
\end{aligned}
$$

where the Fourier series coefficients $a_{m n}$ and $b_{m n}$ are defined in the usual manner. The spatial variation $l_{0}(\xi, \tau)$ in illumination is intended to be uniform and any residual variation is smoothly varying so that $l_{0}$ dominates the power and within $l^{\text {inf }}(x, y)$, the power is concentrated in the first few harmonics. Specifically, we assume that $l_{0}>>l^{\text {inf }}(x, y)$. In reality, however, the illumination on the plate is space limited and can be obtained by multiplying the infinite length illumination with a rectangular function $l(x, y)=$ $l^{\text {inf }}(x, y) \operatorname{rect}(x / W, y / H)$. The space limited $l(x, y)$ can be trans-

\footnotetext{
${ }^{3}$ Under the assumption that the number of tiles over the span of the plate is large, $l_{f}^{\text {inf }}(x, y)$ is also an approximation to $l_{f}(x, y)$.
}

formed into the frequency domain as

$$
\begin{aligned}
l(u, v) & =\sum_{m n} c_{m n} \operatorname{sinc}\left[W\left(u-\frac{m}{P_{x}}\right), H\left(v-\frac{n}{P_{y}}\right)\right] \\
& +\sum_{m n} d_{m n} \operatorname{sinc}\left[W\left(u+\frac{m}{P_{x}}\right), H\left(v+\frac{n}{P_{y}}\right)\right],
\end{aligned}
$$

where $c_{m n}=e^{-\jmath \pi(W u+H v)}\left(a_{m n}-\jmath b_{m n}\right) / 2$ and $d_{m n}=c_{m n}^{*}$. Noting that $W>>P_{x}$ and $H>>P_{y}$, from (8) we can now deduce that the illumination variation forms a bandpass signal with energy distributed in narrow regions about harmonics of the 2-D frequency $\left[1 / P_{x}, 1 / P_{y}\right]$.

\subsection{Lighting Variation Correction}

Recall that the full pattern of illumination across the image is given by $l_{f}(x, y)=l_{0}+l(x, y)$, which is a combination of the DC term comprising of the spatially averaged illumination level $l_{0}$ and $\mathrm{AC}$ terms $l(x, y)$ that causes the visible pattern in the mosaic image. Our analysis culminating in the Fourier spectrum expression (8) indicates that $l(x, y)$ forms a bandpass signal with fundamental frequencies centered at $\left[m / P_{x}, n / P_{y}\right] m, n \in(-1,0,1)$. We would like to eliminate the $\mathrm{AC}$ components to eliminate the lighting variation. In order to allow frequency domain separation of the AC components, we linearize the model in (4) by using a logarithmic transform ${ }^{4}$ :

$$
\begin{aligned}
I^{\prime}(x, y) & =\log (I(x, y)) \\
& =\log \left(l_{0} r(x, y)\right)+\log \left(1+\frac{l(x, y)}{l_{0}}\right) \\
& \approx \log \left(I_{0}(x, y)\right)+\frac{l(x, y)}{l_{0}}
\end{aligned}
$$

where $I_{0}(x, y) \stackrel{\text { def }}{=} l_{0} r(x, y)$ represents the composite image free from lighting variations and the approximation in the last step is due to the fact that the amplitude of the lighting variation $l(x, y)$ is much smaller than the average illumination level $l_{0}$.

The linearlized model of (9) along with the conclusions drawn from our representation of the $\mathrm{AC}$ component of the illumination pattern in (8), immediately suggests that filtering to eliminate the frequencies corresponding to the band pass components in (8) will eliminate the objectionable lighting variation from (9) allowing recovery of an image without the variation by inverting the logarithmic transformation. Specifically, this operation can be written as

$$
I_{\text {rec }}(x, y)=e^{\mathcal{F}^{-1}\left(\mathcal{F}\left(I^{\prime}(x, y)\right) \cdot H(u, v)\right)}
$$

where $\mathcal{F}$ and $\mathcal{F}^{-1}$ represent the operators for Fourier and inverse Fourier transforms, respectively, and $H(u, v)$ is a two-dimensional band-reject filter designed to eliminate the regions in which power is concentrated in the bandpass signal $l(x, y)$, i.e. in narrow regions in the vicinity of the the frequencies $\left.\left[m / P_{x}\right), n / P_{y}\right]$.

In practice, to handle the large size image, we implement this correction via a computationally efficient multiscale approach that reduces the computational and memory requirements. We estimate the patterning correction from a downsampled $(1024 \times 810)$ version of the high resolution $(8401 \times 6649)$ mosaic image ${ }^{5}$. The correction is then upsampled to obtain the correction for the high resolution mosaic. Because the lighting variation is smooth, this methodology causes minimal compromise in performance. Fig. 3(a) illustrates magnitude of the Fourier transform of the (downsampled) mosaic

\footnotetext{
${ }^{4}$ This corresponds to homomorphic processing [3, pp. 259].

${ }^{5}$ Downsampling was performed using the Lanczos filter [4]
} 
image $I^{\prime}(x, y)$, over a subset of the computed frequency plane (that ranges over \pm 7.53 cycles/inch $\times \pm 7.06$ cycles/inch). The frequencies corresponding to the patterning can be clearly seen as peaks in the upper left and lower right quadrants. Because the elimination of frequency regions also eliminates corresponding image frequency components, we empirically adjust the widths of the notches at the different harmonics. The notched regions about the fundamental frequencies, illustrated in Fig. 3(b), are selected as a square $1 / P_{x}-L / 2 \leq|u| \leq 1 / P_{x}+L / 2$ and $1 / P_{y}-L / 2 \leq|v| \leq$ $1 / P_{y}+L / 2$ in frequency space where $L$ represents the width of the square region.

Because the mosaic image is a composition of $27 \times 28$ images of the 8.5-by-6.5-inch daguerreotype plate, we calculate $1 / P_{x}$ and $1 / P_{y}$ as $\frac{27}{8.5}=3.18$ and $\frac{28}{6.5}=4.31 \mathrm{inch}^{-1}$, respectively, and set $L$ as 0.70 inch $^{-1}$ in physical units based on the best visual tradeoff between removing the visible pattern and preserving the relevant image content. The corresponding parameters in the discrete time frequency domain (DTFT) for $1 / P_{x}, 1 / P_{y}$, and $L$ can be calculated using the relation between DTFT and continuous time Fourier transform in [5], which results in multiplying the frequency in physical units by 8.5 when $1024 \times 1024 \mathrm{fft}$ of the mosaic image is taken in this case.

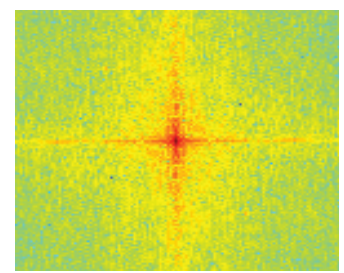

(a) $\mathcal{F}\left(I^{\prime}(x, y)\right)$

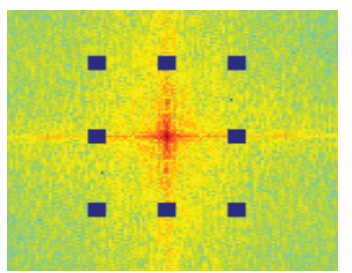

(b) $\mathcal{F}\left(I^{\prime}(x, y)\right) H(u, v)$
Fig. 3. Fourier transform of $I^{\prime}(x, y)$ and notched regions in the vicinity of the primary frequencies.

The recovered image for plate 2 using our proposed method ${ }^{6}$ is shown in Fig. 4(a). Note that in comparison with the original image of Fig. 2 the objectionable pattern of lighting variation is largely eliminated in Fig. 4(a). Using (10) and (11), we can also estimate the patterning correction Fig. 2.

$$
I_{\text {pattern }}(x, y)=I_{r e c}(x, y)-I(x, y),
$$

which is shown in Fig 4(b).

\section{CONCLUSION AND DISCUSSION}

The signal processing analysis and the algorithm that we present in this paper provides a computationally simple and effective method for correction of illumination non-uniformity in stitched mosaics obtained by microscopic capture of daguerreotype images. Applied to the Cincinnati Waterfront Daguerreotype Panorama, the method offers a significant improvement by correcting the illumination patterning and yields a much better rendering of this historically and culturally significant record. The application is one example illustrating how simple signal processing techniques can be readily applied in real-world problems in art and museum imaging applications. Beyond the correction of patterning uniformity, many interesting signal and image processing problems remain to be addressed

\footnotetext{
${ }^{6}$ Other plates yield similar results.
}

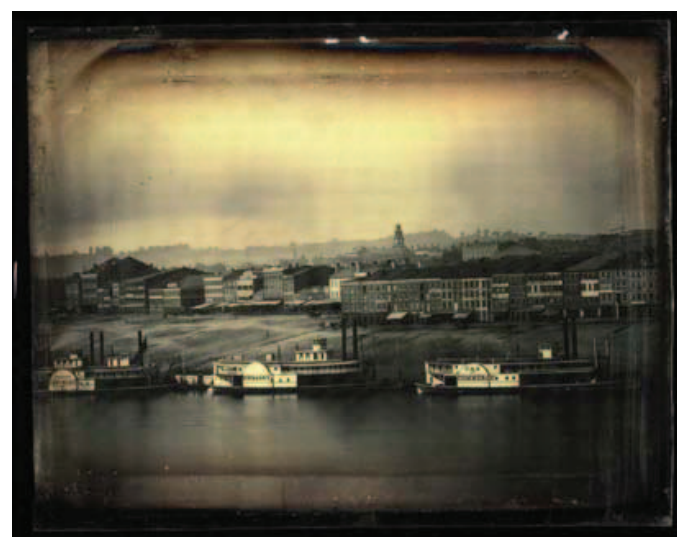

(a) Recovered image $I_{r e c}(x, y)$

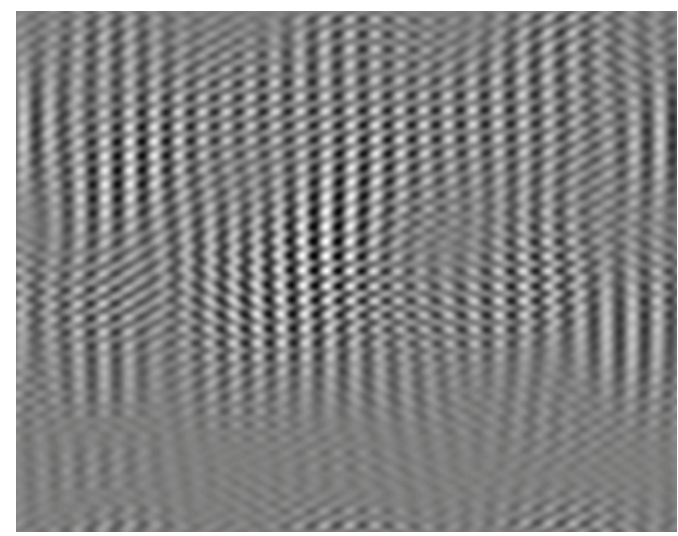

(b) Patterning correction $I_{\text {pattern }}(x, y)$ (scaled)

Fig. 4. Recovered image and the estimated patterning correction.

in this domain and are being investigated in continuing work [6]. We hope this manuscript will also attract additional DSP practitioners to this under-explored area.

\section{REFERENCES}

[1] J. Rehmeyer, "1848 daguerreotypes bring middle america's past to life," Wired, vol. 18, no. 8, Aug. 2010. [Online]. Available: http://www.wired.com/magazine/2010/07/ff_ daguerrotype panorama/

[2] B. Oztan and G. Sharma, "Continuous phase-modulated halftones," IEEE Trans. Image Proc., vol. 18, no. 12, pp. 2718-2734, Dec. 2009.

[3] A. K. Jain, Fundamentals of Digital Image Processing. Englewood Cliffs, N.J.: Prentice-Hall, 1989.

[4] C. Duchon, "Lanczos filtering in one and two dimensions," Journal of Applied Meteorology, vol. 18, no. 8, pp. 1016-1022, 1979.

[5] A. V. Oppenheim and R. W. Schafer, Digital Signal Processing. Englewood Cliffs, N.J.: Prentice-Hall, 1975.

[6] M. Barni, J.-A. Beraldin, C. Lahanier, and A. Piva, "Signal processing in visual cultural heritage [from the guest editors]," Signal Processing Magazine, IEEE, vol. 25, no. 4, pp. 10 -12, july 2008. 